\title{
WEIGHTED METRIC MULTIDIMENSIONAL SCALING
}

\author{
Michael Greenacre \\ Departament d'Economia i Empresa \\ Universitat Pompeu Fabra \\ Ramon Trias Fargas, 25-27 \\ E-08005 Barcelona \\ E-mail: michael@upf.es
}

\section{SUMMARY}

This paper establishes a general framework for metric scaling of any distance measure between individuals based on a rectangular individuals-by-variables data matrix. The method allows visualization of both individuals and variables as well as preserving all the good properties of principal axis methods such as principal components and correspondence analysis, based on the singular-value decomposition, including the decomposition of variance into components along principal axes which provide the numerical diagnostics known as contributions. The idea is inspired from the chi-square distance in correspondence analysis which weights each coordinate by an amount calculated from the margins of the data table. In weighted metric multidimensional scaling (WMDS) we allow these weights to be unknown parameters which are estimated from the data to maximize the fit to the original distances. Once this extra weight-estimation step is accomplished, the procedure follows the classical path in decomposing a matrix and displaying its rows and columns in biplots.

Keywords. biplot, correspondence analysis, distance, multidimensional scaling, singular-value decomposition.

JEL codes. C19, C88

This work was prepared originally for the meeting of the Italian Classification and Data Analysis Group (CLADAG) meeting in Bologna, September 2003, and presented under the title "Weighted Metric Biplots" at the German Classification meeting in Dortmund, March 2004. The invitations of both the above-mentioned societies is gratefully acknowledged as well as the research support of the Spanish Ministry of Science and Technology, grant number BFM2000-1064. 


\section{Introduction}

We are concerned here with methods that transform a rectangular data matrix into a graphical representation of the rows (usually individuals, or subjects) and columns (usually variables, or objects). A typical example of a visualization is the biplot (Gabriel, 1971; Gower \& Hand, 1996) in which a distance approximation is achieved with respect to the individuals, while the variables are depicted by arrows defining biplot axes allowing estimation of the original data values.

An example is shown in Figure 1, where data on 12 countries and five variables on different scales are mapped to a biplot where (squared) distances between countries are standardized Euclidean distances of the form:

$$
d^{2}\left(\mathbf{x}_{i}, \mathbf{x}_{j}\right)=\left(\mathbf{x}_{i}-\mathbf{x}_{j}\right)^{\top} \mathbf{D}_{S}^{-1}\left(\mathbf{x}_{i}-\mathbf{x}_{j}\right)=\sum_{k}\left(x_{i k}-x_{j k}\right)^{2} / s_{k}
$$

where $\mathbf{x}_{i}$ and $\mathbf{x}_{j}$ are the $i$ th and $j$ th rows of the matrix and $\mathbf{D}_{s}$ is the diagonal matrix of standard deviations $s_{k}$. The factors $1 / s_{k}$ are standardizing factors which can alternatively be regarded as weights assigned to each variable in the calculation of the distance between countries. In correspondence analysis (CA) of a table of frequencies we have a similar distance function, where the weights for the rows and the columns in the chi-square distance function are proportional to the inverses of the corresponding row and column margins of the table.

In general we can define the weighted Euclidean (squared) distance:

$$
d^{2}\left(\mathbf{x}_{i}, \mathbf{x}_{j}\right)=\left(\mathbf{x}_{i}-\mathbf{x}_{j}\right)^{\top} \mathbf{D}_{w}\left(\mathbf{x}_{i}-\mathbf{x}_{j}\right)=\sum_{k} w_{k}\left(x_{i k}-x_{j k}\right)^{2}
$$

where $\mathbf{D}_{w}$ contains weights $w_{k}$, to be determined by a process still to be described.

In several contexts, the practitioner is more interested in distance measures which are nonEuclidean. A good example of this is in ecological studies where the data are species-abundances at different sites where equal area- or volume-sampling has been conducted. In this case, ecologists almost always prefer the Bray-Curtis dissimilarity measure, since it has an immediate and simple interpretation, with values from 0 (exactly the same species composition) to 100 (no species in common at all). The Bray-Curtis index $d_{i j}$ between samples $i$ and $j$ with species abundances denoted by $\left\{n_{i k}\right\}$ and $\left\{n_{j k}\right\}$ is defined as follows:

$$
d_{i j}=100 \frac{\sum_{k}\left|n_{i k}-n_{j k}\right|}{\sum_{k} n_{i k}+\sum_{k} n_{j k}}
$$




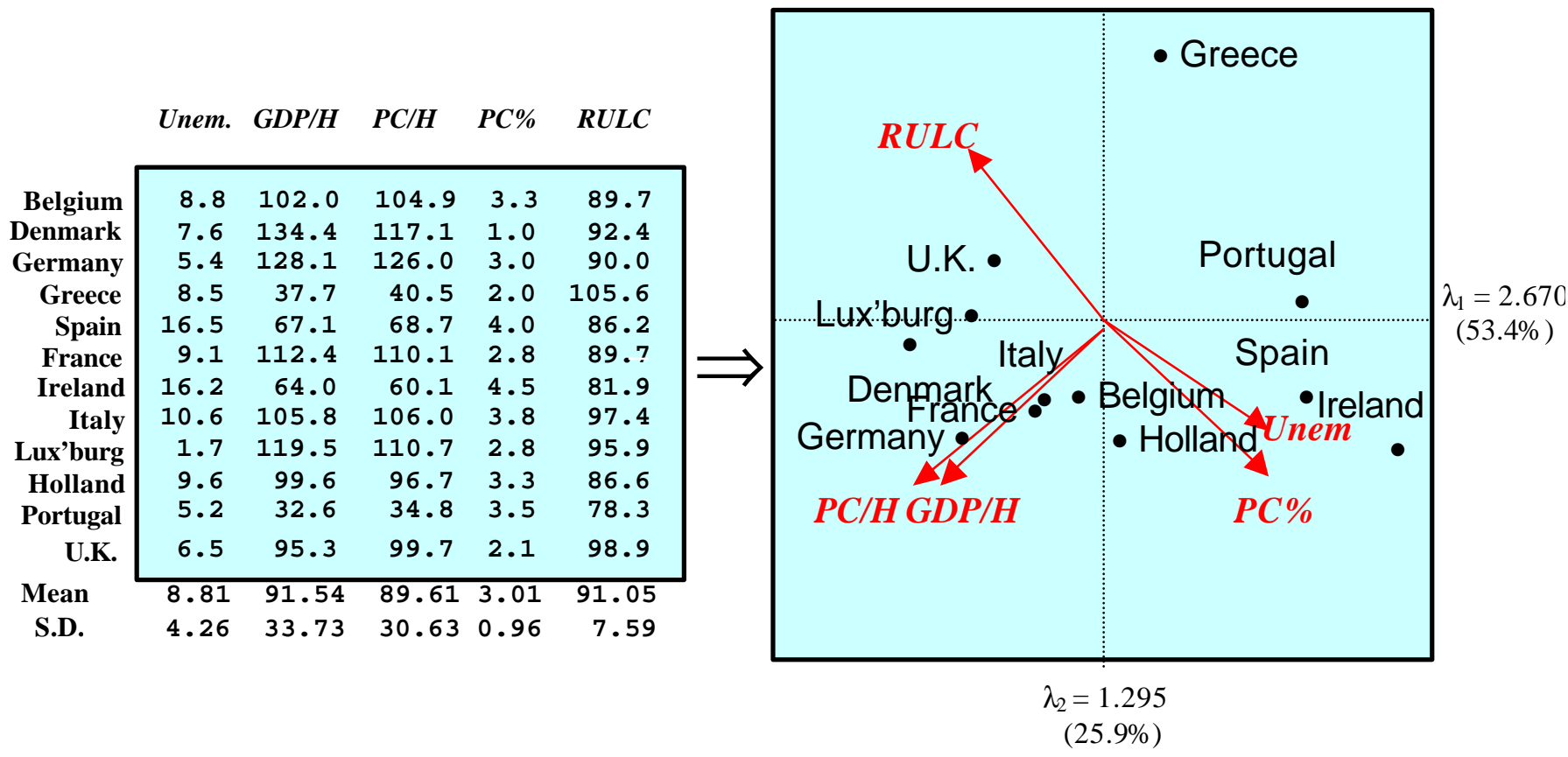

Figure 1: Data matrix on 12 European community countries in 1990, showing five economic indicators: Unemployment Rate (\%), Gross Domestic Product per Head (index), Private Consumption per Head (index), Change in Private Consumption (\%) and Real Unit Labour Cost (index). The principal component biplot on standardized data is shown on the right, with vectors indicating biplot axes for each variable.

Such a dissimilarity measure is simple to understand, but non-Euclidean (see Gower \& Legendre, 1986). Often nonmetric MDS is used to analyse these indices (see, for example, Field, Warwick \& Clarke, 1982), but our interest here is in metric MDS since there are many relevant spin-offs in the classical metric approach, most importantly the natural biplot framework thanks to the singular value decomposition, as well as the convenient breakdown of variance across principal axes of both the rows and columns which provide useful numerical diagnostics in the interpretation and evaluation of the results. The idea will be to approximate the distances of choice, however they are defined, by a weighted Euclidean distance. The weights estimated in this process will be those that are inherently assigned to the variables by the chosen distance function.

In Section 2 we shall summarize the classical MDS framework with weights. Then in Section 3 we describe how any distance measure between individuals can be approximated by a weighted Euclidean metric. In Section 4 we give some examples of this approach and conclude with a discussion in Section 5. 


\section{Weighted Multidimensional Scaling}

Our main interest is in weighting the variables in the definition of distances between the individuals, but exactly the same technology allows weighting of the individuals as well to differentiate their effect on determining the eventual solution space. Since the weighting of the individuals serves a different purpose from the weighting of the variables, we shall use the terms mass for an individual and weight for a variable (in correspondence analysis the term mass is used exactly in the sense used here). Both individual masses and variable weights will be included in our description that follows. This description is essentially that of the geometric definition of correspondence analysis (see Greenacre, 1984, chapter 2), the only difference being that the weights on the variables are unknown, to be determined, and not prescribed.

Suppose that we have a data matrix $\mathbf{Y}(n \times m)$, usually pre-centred with respect to rows or columns or both. Let $\mathbf{D}_{r}(n \times n)$ and $\mathbf{D}_{w}(m \times m)$ be diagonal matrices of row (individual) masses and column (variable) weights respectively. With no loss of generality the row masses are presumed to have a sum of 1. The rows of $\mathbf{Y}$ are presumed to be points in an $m$-dimensional Euclidean space, structured by the inner product and metric defined by the weight matrix $\mathbf{D}_{w}$. The solution, a lowdimensional subspace which fits the points as closely as possible, is established by weighted leastsquares, where each point is weighted by its mass. The following function is thus minimized:

$$
\operatorname{In}(\mathbf{Y}-\hat{\mathbf{Y}})=\sum_{i} r_{i}\left(\mathbf{y}_{i}-\hat{\mathbf{y}}_{i}\right)^{\mathrm{T}} \mathbf{D}_{w}\left(\mathbf{y}_{i}-\hat{\mathbf{y}}_{i}\right)
$$

where $\hat{\mathbf{y}}_{i}$, the $i$-th row of $\hat{\mathbf{Y}}$, is the closest low-dimensional approximation of $\mathbf{y}_{i}$. The function $\operatorname{In}(*, *)$ stands for the inertia, in this case the inertia of the difference between the original and approximated matrices. The total inertia, which is being decomposed or "explained" by the solution, is equal to $\mathrm{I}(\mathbf{Y})$.

As is well-known (see, for example, Greenacre, 1984, Appendix), the solution can be obtained neatly using the generalized singular value decomposition (GSVD) of the matrix $\mathbf{Y}$. Computationally, using an ordinary SVD algorithm, the steps in finding the solution are to first preprocess the matrix $\mathbf{Y}$ by pre- and post-multiplying by the square roots of the weighting matrices, then calculate the SVD and then post-process the solution using the inverse transformation to obtain principal and standard coordinates. The steps are summarized as follows:

$$
\text { 1. } \mathbf{S}=\mathbf{D}_{r}^{1 / 2} \mathbf{Y D}_{w}^{1 / 2}
$$




\section{2. $\mathbf{S}=\mathbf{U D}_{\alpha} \mathbf{V}^{\top}$}

3. Principal coordinates of rows: $\quad \mathbf{F}=\mathbf{D}_{r}^{-1 / 2} \mathbf{U D}_{\alpha}$

4. Standard coordinates of columns: $\mathbf{G}=\mathbf{D}_{w}^{-1 / 2} \mathbf{V}$

The columns (variables) are conventionally depicted by arrows and the rows (individuals) by points. A two-dimensional solution, say, would use the first two columns of $\mathbf{F}$ and $\mathbf{G}$. The total inertia is the sum of squares of the singular values $\alpha_{1}^{2}+\alpha_{2}^{2}+\ldots$, the inertia accounted for in two-dimensional solution is the sum of the first two terms $\alpha_{1}^{2}+\alpha_{2}^{2}$ while the inertia not accounted for (formula (4)) is the remainder of the sum: $\alpha_{3}^{2}+\alpha_{4}^{2}+\ldots$.

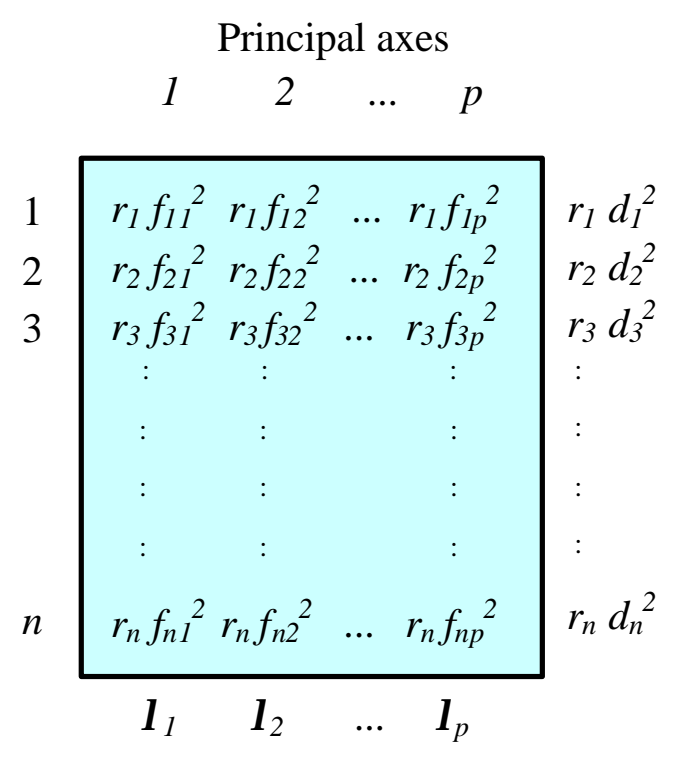

Table 1: Decomposition of inertia of $n$ row points along all $p$ principal axes of the matrix. Each row adds up to the inertia of a point, the mass $\left(r_{i}\right)$ times squared distance $\left(d_{i}{ }^{2}\right)$ of the point to the centre, while each column adds up the principal inertia $\lambda_{k}=\alpha_{k}^{2}$ of the corresponding axis. Rows and columns expressed relative to their respective totals constitute the contributions, or numerical diagnostics used to support the interpretation of the solution space.

Apart from this simple decomposition of the variance in the data matrix, there is another benefit of the least-squares approach via the SVD, namely a further breakdown of inertia for each point along each principal axis. Since this decomposition applies to points in principal coordinates, we show it for the row points in Table 1 (a similar decomposition can be shown for column points in principal coordinates by merely scaling the standard coordinates by their respective singular values). 


\section{Computing the Variable Weights}

We now consider the case when a general distance function is used to measure distance or dissimilarity between individuals, not necessarily a Euclidean-imbeddable distance. Using conventional MDS notation let us suppose that $\delta_{i j}{ }^{2}$ is the observed dissimilarity between individuals $i$ and $j$ based on their description vectors $\mathbf{x}_{i}$ and $\mathbf{x}_{j}$. We use $d_{i j}{ }^{2}=d_{i j}{ }^{2}(\mathbf{w})$ to indicate the weighted Euclidean distance based on (unknown) weights in the vector $\mathbf{w}$. The problem is then to find the weights which give the best fit to the observed dissimilarities, either minimizing fit to distances (least-squares scaling, or LSS) or to squared distances (least-squares squared scaling, or LSSS). As always it is easier to fit to squared distances, which is the approach we take here. Thus the objective function is:

$$
\operatorname{minimize} \sum_{i} \sum_{j}\left(\delta_{i j}^{2}-d_{i j}^{2}(\mathbf{w})\right)^{2} \quad \text { over all } \mathbf{w} \geq 0
$$

that is,

$$
\operatorname{minimize} \sum_{i} \sum_{j}\left(\delta_{i j}^{2}-\sum_{k} w_{k}\left(x_{i k}-x_{j k}\right)^{2}\right)^{2} \text { over all } \mathbf{w} \geq 0
$$

Ignoring for the moment the non-negativity restriction on $\mathbf{w}$, the problem can be solved by leastsquares regression without a constant as follows:

- Define $\delta=\operatorname{vec}\left(\delta_{i j}{ }^{2}\right)$ as the $1 / 2 n(n-1)$ vector of given squared distances, that is the half-triangle of distances strung out as a vector.

- Define $\mathbf{X}=\left[\left(x_{i k}-x_{j k}\right)^{2}\right]$ as the $1 / 2 n(n-1) \times m$ matrix of squared differences between the values of a variable, for each pair of individuals.

- Fit the multiple regression model $\delta=\mathbf{X w}+\mathbf{e}$ which has least-squares solution $\mathbf{w}=\left(\mathbf{X}^{\top} \mathbf{X}\right)^{-1} \delta$.

In our experience it frequently occurs that the weights calculated without constraints turn out to be positive. However, when this is not the case, minimisation has to be performed with constraints:

$$
\operatorname{minimize}(\boldsymbol{\delta}-\mathbf{X w})^{\top}(\delta-\mathbf{X w}) \text { subject to } \mathbf{w} \geq 0
$$


This is a quadratic programming problem (see, for example, Bartels, Golub \& Saunders, 1970) which can be solved with standard software, for example function nlregb in S-PLUS (1999) see also www.numerical.rl.ac.uk/qp/qp.html.

In the regression described above the masses assigned to the individuals can be taken into account by performing weighted least-squares regression, with the weights assigned to each $(i, j)$-th element equal to the product $r_{i} r_{j}$ of the corresponding masses. That is, define the $1 / 2 n(n-1) \times 1 / 2 n(n-1)$ diagonal matrix $\mathbf{D}_{r r}$ with these products down the diagonal and then minimize the quadratic form $(\delta-\mathbf{X w})^{\top} \mathbf{D}_{r r}(\boldsymbol{\delta}-\mathbf{X w})$, which in the unconstrained case gives solution $\mathbf{w}=\left(\mathbf{X}^{\top} \mathbf{D}_{r r} \mathbf{X}\right)^{-1} \mathbf{D}_{r r} \delta$.

The goodness of fit of the weighted Euclidean distances to the original distances can be measured by the usual coefficient of determination $R^{2}$. Our visualization of the original data matrix passes through two stages of approximation, first the fitting of the distances by estimating the variable weights, and second the matrix approximation of the GSVD to give the graphical display of the weighted Euclidean distances and the associated biplot vectors for the variables.

\section{Application: Bhattacharyya (arc cos) distance}

This research was originally inspired by an article in the Catalan statistical journal Qüestiio by Vives \& Villaroya (1996), who apply Intrinsic Data Analysis (Rios, Villaroya \& Oller, 1994) to visualize in the form of a biplot a compositional data matrix, specifically the composition in each of the 41 Catalan counties (comarques) of eight different professional groups (the full table is given in the appendix). This analysis is based on the Bhattacharyya distance between counties:

$$
d^{2}\left(\mathbf{p}_{i}, \mathbf{p}_{j}\right)=\arccos \left(\sum_{k} \sqrt{p_{i k} p_{j k}}\right)
$$

where the function arc $\cos$ is the inverse cosine. The same authors report that their results are almost identical to those of correspondence analysis. Applying weighted MDS to the same data the weights are estimated to be the following for the eight professional groups: 


\section{Weights estimated by fitting to Bhattacharyya distances}

Pro\&Tec PersDir ServAdm Com\&Ven Hot\&Alt Agr\&Pes Indust ForArm
1.9
4.6
5.7
1.9
2.0
1.6
$0.9 \quad 41.1$

\section{Weights implied by correspondence analysis $\left(1 / c_{k}\right)$}

Pro\&Tec PersDir ServAdm Com\&Ven Hot\&Alt Agr\&Pes Indust ForArm

$\begin{array}{llllllll}9.6 & 49.4 & 8.8 & 8.5 & 10.0 & 8.1 & 2.4 & 263.0\end{array}$

Figure 2: Comparison of estimated weights to fit optimally to arc cos distances and correspondence analysis weights.

It is interesting to see that the variable "ForArm" (forces armades in Catalan, i.e. armed forces) receives much higher weight than the others, very similar to the situation in CA where it is weighted highly because of very low relative frequency and thus low variance. The arc cos distance inherently weights this variable highly as well even though this is not at all obvious from its definition in (10).

The fit of the weighted Euclidean distances to the arc cos distances is excellent: sum-of-squared distances, SSD = 9.570, with sum-of-squares due to regression, SSR $=9.327$ (97.5\%) and sum-ofsquares due to error, $\mathrm{SSE}=0.243(2.5 \%)$.

In Figure 3 we see the form biplot of the results. The form biplot scales the rows (counties) in principal coordinates so that we can interpret the inter-row distances, and the columns (professional categories) in standard coordinates. Projecting the rows onto the biplot axes defined by the column vectors will give an approximation to the original percentages in the data matrix. The alternative is 
to plot the results as a covariance biplot where the rows are in standard coordinates and the columns are in principal coordinates, in which case the covariance structure amongst the columns is displayed.

Finally, in Table 2 we have the contributions to inertia that are the spin-off of our approach - we show the contributions for the column points. The columns of Table 1 relative to their sums (the principal inertias, or squared singular values) are given in the columns headed CTR, for each of the two dimensions, often called the absolute contributions in correspondence analysis. The rows of Table 1 relative to their sums (the inertias of the column points) are given in the columns headed COR. 


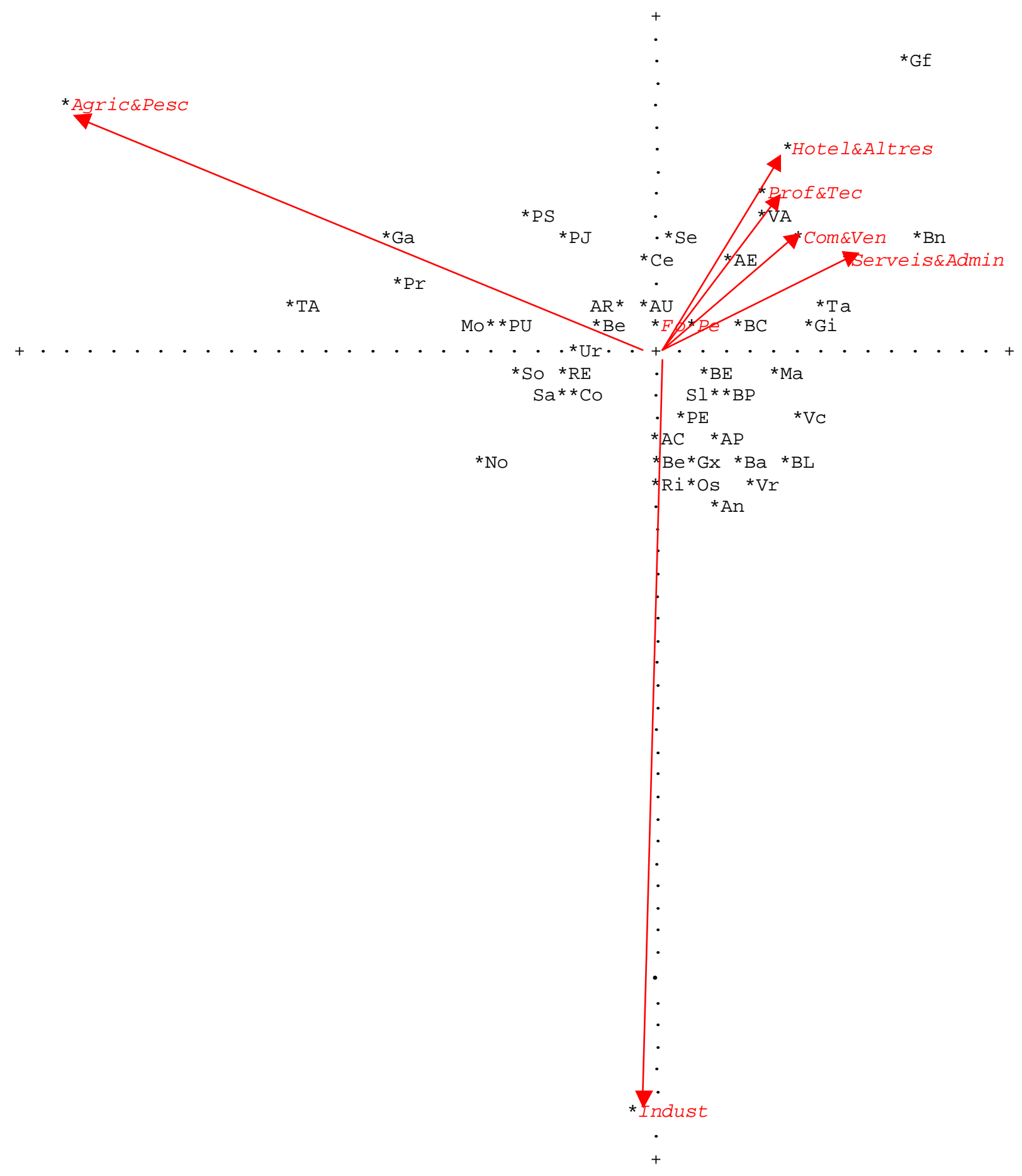

Figure 3: Form biplot of 41 Catalan counties (in principal coordinates) and 8 professional categories (in standard coordinates). 


\begin{tabular}{|c|c|c|c|c|c|}
\hline & Quality & & Princ & al ax & \\
\hline & & & 1 & & \\
\hline & $\mathrm{QLT}$ & CTR & $\mathrm{COR}$ & CTR & $\mathrm{COR}$ \\
\hline Prof\&Tec & 622 & 24 & 304 & 46 & 318 \\
\hline PersDir & 385 & 5 & 308 & 2 & 77 \\
\hline Serveis\&Admin & 832 & 243 & 754 & 46 & 78 \\
\hline Com\&Ven & 772 & 47 & 604 & 24 & 168 \\
\hline Hotel\&Altres & 608 & 41 & 280 & 89 & 328 \\
\hline Agric\&Pesc & 989 & 636 & 907 & 105 & 82 \\
\hline Indust & 998 & 0 & 1 & 676 & 997 \\
\hline ForArm & 148 & 0 & 0 & 8 & 148 \\
\hline $\begin{array}{l}\text { Principal inertia } \\
\text { (\% of total) }\end{array}$ & & $\begin{array}{l}0 . \\
(57\end{array}$ & $\begin{array}{l}203 \\
1 \%)\end{array}$ & $\begin{array}{l}0 . \\
(31\end{array}$ & $\begin{array}{l}111 \\
1 \%)\end{array}$ \\
\hline
\end{tabular}

Table 2 Decomposition of inertia of 8 column points along first two principal axes. The principal inertias (eigenvalues, or squared singular values) are decomposed amongst the points as given in the columns CTR, given in "permills", for example the first axis is determined mostly by points Agric\&Pesc $(63.6 \%)$ and ServAdm (24.3\%). These are the columns of Table 1 relative to their totals. The inertia of a point is decomposed along the principal axes according to the values in the columns COR. These are the rows of Table 1 relative to their totals, and are also squared correlations (angle cosines) of the points with the principal axes. Thus the point Indust is mostly explained by the second axis, while ForArm is not well explained by either axis and also plays hardly any role in determining the twodimensional solution, even with the large weight assigned to it. The column QLT refers to quality of display in the plane, and is the sum of the COR columns. 


\section{References}

Bartels, R.H., Golub, G.H. \& Saunders, M.A. (1970) Numerical techniques in mathematical programming. In Nonlinear Programming (eds J.B. Rosen, O.L. Mangasarian \& K. Ritter), London: Academic Press, pp. 123-176.

Field, J.G., Clarke, K.R. \& Warwick, R.M. (1982) A practical strategy for analysing multispecies distribution patterns. Marine Ecology Progress Series, 8, 37-52.

Gabriel, K. R. (1971) The biplot-graphic display of matrices with applications to principal component analysis. Biometrika, 58, 453-467.

Gower, J.C. \& Legendre, P. (1986) Metric and Euclidean properties of dissimilarity coefficients. Journal of Classification, 3, 5-48.

Greenacre, M.J. (1984) Theory and Applications of Correspondence Analysis. London: Academic Press.

Rios, M., Villaroya, A. \& Oller, J.M. (1994) Intrinsic data analysis : a method for the simultaneous representation of populations and variables. Research report 160, Department of Statistics, University of Barcelona.

Vives, S. \& Villaroya, A. (1996) La combinació de tècniques de geometria diferencial amb anàlisi multivariant clàssica: una aplicació a la caracterització de les comarques catalanes. Qüestiio, 20, 449-482.

S-PLUS (1999). S-PLUS 2000 Guide to Statistics, Volume 1, Mathsoft, Seattle, WA. 


\section{Appendix}

Percentages of different professional groups in the 41 Catalan counties

\begin{tabular}{|c|c|c|c|c|c|c|c|c|c|}
\hline COUNTY & $\begin{array}{r}\text { Prof./ } \\
\text { TeC. }\end{array}$ & $\begin{array}{l}\text { Pers. } \\
\text { Dir. }\end{array}$ & $\begin{array}{l}\text { Serveis } \\
\text { admin. }\end{array}$ & $\begin{array}{l}\text { Comerc. } \\
\text { Vened. }\end{array}$ & $\begin{array}{l}\text { Hotel. } \\
\text { altres }\end{array}$ & $\begin{array}{l}\text { A gric. } \\
\text { Pesc. }\end{array}$ & Indust. & $\begin{array}{c}\text { Forces } \\
\text { arm. }\end{array}$ & total \\
\hline (AC) Alt Camp & 9.6 & 1.9 & 11.3 & 11.1 & 6.8 & 9.9 & 49.1 & 0.2 & 100 \\
\hline (AE) Alt Emporda & 8.4 & 2.3 & 14.4 & 15.7 & 13.8 & 10.0 & 34.5 & 0.9 & 100 \\
\hline (AP) Alt Pened\&s & 9.1 & 1.9 & 13.8 & 11.5 & 7.5 & 6.9 & 49.2 & 0.1 & 100 \\
\hline (AU) Alt Urgell & 10.4 & 1.8 & 11.1 & 13.6 & 10.7 & 14.3 & 37.1 & 1.1 & 100 \\
\hline (AR) Alta Ribagorea & 13.9 & 1.8 & 7.8 & 10.4 & 15.8 & 12.9 & 37.3 & 0.1 & 100 \\
\hline (An) A noia & 8.8 & 2.0 & 11.0 & 11.3 & 7.7 & 3.6 & 55.6 & 0.1 & 100 \\
\hline (Ba) Bages & 11.3 & 1.8 & 11.7 & 12.8 & 8.2 & 3.2 & 50.8 & 0.3 & 100 \\
\hline ) Baix Camp & 12.1 & 2.1 & 13.1 & 15.0 & 11.1 & 7.0 & 39.3 & 0.2 & 100 \\
\hline (Be) Baix Ebre & 10.9 & 1.7 & 10.3 & 12.5 & 8.8 & 16.3 & 39.3 & 0.3 & 100 \\
\hline (BE) Baix Emporda & 8.2 & 2.2 & 10.9 & 14.3 & 13.6 & 8.0 & 42.5 & 0.4 & 100 \\
\hline (BL) B aix L lobregat & 5.8 & 1.9 & 14.7 & 12.6 & 11.7 & 1.2 & 52.0 & 0.1 & 100 \\
\hline (BP) Baix Penedss & 7.9 & 2.3 & 12.1 & 14.2 & 12.6 & 5.6 & 44.9 & 0.3 & 100 \\
\hline 3 arcelona & 17.1 & 2.9 & 21.4 & 14.8 & 11.2 & 0.4 & 32.1 & 0.1 & 100 \\
\hline$(\mathrm{Be})$ & 10.1 & 1.2 & 8.9 & 11.5 & 8.3 & 8.3 & 51.0 & 0.6 & 100 \\
\hline (Ce) Cerdanya & 10.0 & 2.3 & 9.4 & 13.8 & 15.9 & 13.6 & 34.3 & 0.8 & 100 \\
\hline (Co) Conca de Barbera & 8.6 & 1.9 & 9.7 & 9.7 & 7.5 & 16.3 & 46.2 & 0.1 & 100 \\
\hline arraf & 12.8 & 2.0 & 12.6 & 14.2 & 13.1 & 3.1 & 42.1 & 0.2 & 100 \\
\hline (Ga) Garrigues & 7.9 & 1.2 & 7.7 & 9.1 & 6.2 & 34.3 & 33.5 & 0.2 & 100 \\
\hline txa & 10.1 & 2.1 & 11.0 & 10.8 & 7.5 & 6.7 & 51.6 & 0.2 & 100 \\
\hline Giror & 14.2 & 2.3 & 17.2 & 13.9 & 9.9 & 3.3 & 38.6 & 0.5 & 100 \\
\hline (M a) M aresma & 11.9 & 3.2 & 13.9 & 14.4 & 10.0 & 4.2 & 42.3 & 0.2 & 100 \\
\hline (Mo) M ontsia & 7.0 & 1.5 & 8.4 & 10.8 & 7.3 & 24.1 & 40.5 & 0.4 & 100 \\
\hline guera & 7.3 & 1.2 & 6.0 & 7.9 & 5.3 & 20.8 & 51.2 & 0.2 & 100 \\
\hline (Os) Osona & 9.9 & 1.8 & 10.7 & 11.0 & 6.6 & 6.2 & 53.6 & 0.1 & 100 \\
\hline (PJ) Pallar & 12.4 & 1.7 & 10.4 & 10.1 & 8.9 & 20.8 & 33.4 & 2.2 & 100 \\
\hline Palla & 13.4 & 1.3 & 9.6 & 7.1 & 14.7 & 23.8 & 29.7 & 0.3 & 100 \\
\hline (PU) Pla d'Urgell & 8.2 & 1.6 & 9.7 & 9.7 & 5.7 & 24.6 & 40.1 & 0.2 & 100 \\
\hline (PE) Pla de l'E stany & 10.9 & 2.2 & 12.3 & 10.5 & 7.0 & 9.5 & 47.5 & 0.1 & 100 \\
\hline (Pr) Priorat & 8.7 & 1.0 & 7.4 & 7.7 & 7.0 & 32.2 & 35.7 & 0.3 & 100 \\
\hline (RE) Ribera d'Ebre & 12.4 & 1.0 & 9.1 & 8.7 & 7.8 & 17.5 & 43.2 & 0.4 & 100 \\
\hline Ripolles & 9.2 & 1.8 & 8.3 & 10.1 & 9.2 & 7.3 & 53 & 0.2 & 100 \\
\hline garra & 9.9 & 1.9 & 9.9 & 8.5 & 6.3 & 17 & 45 & 0.1 & 100 \\
\hline (Se) Segria & 13.0 & 2.1 & 13.8 & 13.8 & 10.4 & 14.4 & 31.5 & 1.0 & 100 \\
\hline (SI) Selva & 7.3 & 2.0 & 10.8 & 12.5 & 15.2 & 5.7 & 46.4 & 0.2 & 100 \\
\hline (So) Solsones & 10.2 & 1.4 & 7.8 & 7.4 & 8.2 & 21.2 & 43.7 & 0.1 & 100 \\
\hline (Ta) Tarragones & 14.2 & 2.1 & 16.6 & 12.9 & 12.9 & 2.9 & 37.7 & 0.6 & 100 \\
\hline Terra A Ita & 4.8 & 0.9 & 4.9 & 7.2 & 4.7 & 39.1 & 38.1 & 0.4 & 100 \\
\hline$(\mathrm{Ur})$ & 9.1 & 2.1 & 9.8 & 12.7 & 6.7 & 17.7 & 41.7 & 0.3 & 100 \\
\hline (VA & 11.2 & 6.9 & 10.8 & 13.6 & 21.3 & 5.4 & 29.5 & 1.2 & 100 \\
\hline (Vc) Valles Occidental & 12.1 & 2.3 & 14.6 & 13.2 & 9.0 & 0.7 & 48.1 & 0.1 & 100 \\
\hline (Vr) Valles Orinetal & 9.3 & 2.2 & 13.2 & 11.3 & 8.2 & 2.4 & 53.2 & 0.1 & 100 \\
\hline average & 12.8 & 2.4 & 16.3 & 13.5 & 10.4 & 3.7 & 40.7 & 0.2 & 100 \\
\hline
\end{tabular}

